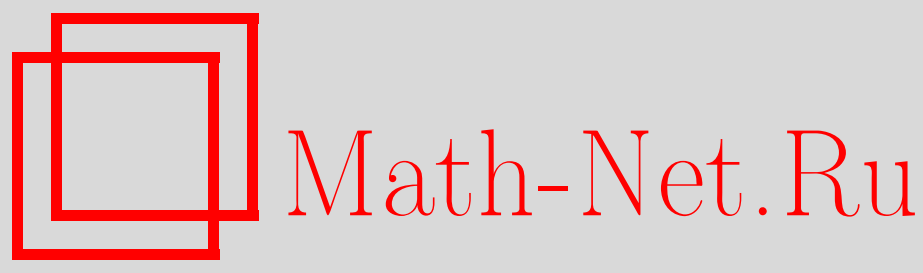

С. А. Кащенко, Регулярные и нерегулярные решения в задаче о дислокациях в твердом теле, ТМФ, 2018, том 195, номер 3, 362-380

DOI: https://doi.org/10.4213/tmf9421

Использование Общероссийского математического портала Math-Net.Ru подразумевает, что вы прочитали и согласны с пользовательским соглашением http://www.mathnet.ru/rus/agreement

Параметры загрузки:

IP : 54.197 .130 .99

26 апреля 2023 г., 18:04:24

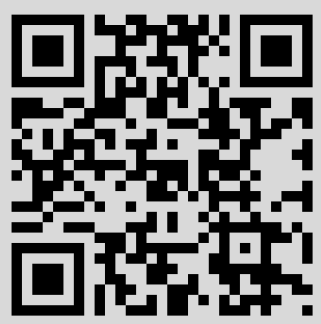




\section{РЕГУЛЯРНЫЕ И НЕРЕГУЛЯРНЫЕ РЕШЕНИЯ В ЗАДАЧЕ О ДИСЛОКАЦИЯХ В ТВЕРДОМ ТЕЛЕ}

Рассмотрены асимптотические по невязке решения исходного дифференциального уравнения с отклонениями пространственной переменной. Все решения естественным образом разбиваются на классы, регулярно и нерегулярно зависящие от параметров задачи. В различных областях из малой окрестности нулевого состояния равновесия фазового пространства построены специальные нелинейные распределенные уравнения и системы уравнений, зависящие от континуальных семейств некоторых параметров. В частности, показано, что решения исходного пространственного одномерного уравнения можно описать решениями специальных уравнений и систем уравнений шредингеровского типа в пространственно двумерной области изменения аргументов.

Ключевые слова: бифуркации, устойчивость, нормальные формы, сингулярные возмущения, динамика.

DOI: https://doi.org/10.4213/tmf9421

\section{1. ПОСТАНОВКА ЗАДАЧИ}

Пусть имеется простейшая кристаллическая структура, состоящая из слоев атомов, расположенных на некотором расстоянии друг от друга. Френкелем и Конторовой была предложена математическая модель поведения точечного дефекта в кристаллической структуре твердого тела [1]. Эти дефекты иногда называют дислокациями. В настоящее время под дислокацией понимается более сложное несовершенство кристаллической структуры, чем любой из точечных дефектов [2].

Для описания дислокаций в твердом теле в работе [1] предложено уравнение движения $n$-го атома в решетке

$$
m \ddot{y}_{n}+\alpha \sin y_{n}=\beta\left(y_{n+1}-2 y_{n}+y_{n-1}\right), \quad n=1, \ldots, N .
$$

Работа выполнена при финансовой поддержке Министерства образования и науки РФ в рамках государственного задания № 1.12873.2018/12.1.

* Ярославский государственный университет им. П. Г. Демидова, Ярославль, Россия. E-mail: kasch@uniyar.ac.ru

${ }^{\dagger}$ Национальный исследовательский ядерный университет "МИФИ", Москва, Россия 
Здесь $m, \alpha$ и $\beta$ - положительные коэффициенты, а $y_{n}=y_{n}\left(t, x_{n}\right)$ - отклонение $n$-го атома от положения равновесия. Для $y_{n}\left(t, x_{n}\right)$ при крайних значениях $n$ выполнены условия, характерные либо для периодической краевой задачи $y_{N+1}=y_{1}, y_{0}=y_{N}$, либо для задачи Дирихле $y_{N+1}=y_{0}=0$, либо для задачи Неймана $y_{N+1}=y_{N}$, $y_{0}=y_{1}$. Точки $x_{n}$ лежат на отрезке $[0,2 \pi]$, и $x_{n+1}=x_{n}+\varepsilon$, где $\varepsilon=2 \pi N^{-1}$. Предполагается, что $N \gg 1$ или, что то же самое,

$$
0<\varepsilon \ll 1
$$

Переходя к непрерывному распределению масс, из уравнения (1) получаем (после очевидных перенормировок и замены $\alpha \sin y$ на более общую функцию $f(y))$ уравнение

$$
\ddot{y}+f(y)=y(t, x+\varepsilon)-2 y+y(t, x-\varepsilon) .
$$

Для определенности ниже считаем, что выполнены периодические краевые условия

$$
y(t, x+2 \pi) \equiv y(t, x) .
$$

Нелинейную функцию $f(y)$ примем в виде

$$
f(y)=a y+b y^{3}+\varphi(y), \quad \text { где } \quad \varphi(y)=o\left(|y|^{3}\right) \quad \text { при } \quad y \rightarrow 0 .
$$

В статье [1] рассмотрен случай, когда $f(y)=\varepsilon^{2} \sin y$, т. е. $a=\varepsilon^{2}, b=\varepsilon^{2} / 6$. Отметим также работы [3]-[9], в которых рассматривалась близкая к уравнению (1) задача Ферми-Паста-Улама.

Поставим задачу исследования при условии (2) поведения всех решений краевой задачи (3), (4) с начальными условиями из некоторой достаточно малой (и не зависящей от $\varepsilon$ ) окрестности нулевого состояния равновесия. Центральную роль при этом играет поведение решений линеаризованной в нуле краевой задачи

$$
\ddot{y}+a y=y(t, x+\varepsilon)-2 y+y(t, x+\varepsilon), \quad y(t, x+2 \pi) \equiv y(t, x) .
$$

Характеристическое уравнение для задачи (5) имеет вид

$$
\lambda^{2}+a=-4 \sin ^{2}\left(\frac{\varepsilon k}{2}\right), \quad k=0, \pm 1, \ldots
$$

Ниже предполагаем, что параметр $а$ положителен, и для некоторых фиксированных параметров $a_{0}$ и $a_{1}$ записываем соотношение

$$
a=a_{0}+\varepsilon a_{1}, \quad a_{0} \geqslant 0 \quad \text { и } \quad a_{1}>0 \quad \text { при } \quad a_{0}=0 .
$$

Все корни уравнения (6) являются чисто мнимыми. Поэтому в задаче об устойчивости нуля в выражениях (3), (4) реализуется критический случай бесконечной размерности. Используя методы работ [10]-[14], ниже мы строим семейства специальных краевых задач для описания различных групп решений исходной краевой задачи (3), (4). Отметим, что особо важным с прикладной точки зрения [1], [15], [16] является случай, когда параметр $а$ мал. В связи с этим в разделе 2 мы рассматриваем случай, когда $a_{0}=0$, т. е.

$$
a=\varepsilon^{2} a_{1} .
$$


Статья состоит из двух основных разделов. В разделе 2 исследуются регулярные решения, т. е. такие решения, для которых имеет место асимптотическое представление

$$
y(t, x+\varepsilon)=y(t, x)+\varepsilon y^{\prime}(t, x)+\frac{1}{2} \varepsilon^{2} y^{\prime \prime}(t, x)+\cdots .
$$

$\mathrm{C}$ точки зрения расположения корней характеристического уравнения (6) в первом разделе речь идет о решениях, формирующихся в своей основе на модах с "конечными" (т. е. не зависящими от параметра $\varepsilon$ ) значениями номеров $k$.

Во разделе 3 рассматриваются нерегулярные решения, которые содержат быстро осциллирующие составляющие. Они формируются на модах, близких к асимптотически большим (при $\varepsilon \rightarrow 0)$ значениям порядка $\varepsilon^{-1}$. В разделе 4 приведены выводы.

\section{2. РЕГУЛЯРНЫЕ РЕШЕНИЯ}

Здесь мы остановимся на изучении регулярных решений краевой задачи (3), (4). Подставим представление (8) в уравнение (3). Для определения $y(t, x)$ получаем с точностью до $о\left(\varepsilon^{6}\right)$ краевую задачу

$$
\begin{gathered}
\frac{\partial^{2} y}{\partial t^{2}}=\varepsilon^{2}\left[\frac{\partial^{2} y}{\partial x^{2}}+\frac{\varepsilon^{2}}{12} \frac{\partial^{4} y}{\partial x^{4}}+\frac{2 \varepsilon^{4}}{6 !} \frac{\partial^{6} y}{\partial x^{6}}\right]-f(y), \\
y(t, x+2 \pi) \equiv y(t, x) .
\end{gathered}
$$

Отметим, что на таком пути в статье [1] было получено известное уравнение, которое носит название уравнения синус-Гордона. Обратим внимание, что без учета слагаемого $2 \varepsilon^{4}(6 !)^{-1} \partial^{6} y / \partial x^{6}$ краевая задача $(9),(10)$ является некорректной. В частности, не выполняется теорема существования и единственности решений.

Итак, при всех достаточно малых значениях $\varepsilon$ рассматривается вопрос о локальном (в достаточно малой и не зависящей от $\varepsilon$ окрестности нулевого состояния равновесия) поведении всех решений краевой задачи (9), (10).

Важную роль при анализе решений из малой окрестности нуля играет линеаризованное уравнение

$$
\frac{\partial^{2} y}{\partial t^{2}}=\varepsilon^{2}\left[\frac{\partial^{2} y}{\partial x^{2}}+\frac{\varepsilon^{2}}{12} \frac{\partial^{4} y}{\partial x^{4}}+\frac{2 \varepsilon^{4}}{6 !} \frac{\partial^{6} y}{\partial x^{6}}\right]-a y
$$

с краевыми условиями (10). Характеристическое для (10), (11) уравнение записывается как

$$
\lambda^{2}=-\varepsilon^{2}\left((2 \pi k)^{2}-\frac{(2 \pi k)^{4}}{12} \varepsilon^{2}+\frac{2(2 \pi k)^{6}}{6 !} k^{6}\right)-a, \quad k=0, \pm 1, \ldots
$$

Все корни (12) имеют нулевые вещественные части. Это означает, что в задаче об устойчивости нулевого решения реализуется критический случай бесконечной размерности. Такого типа критические случаи исследовались в работах [10]-[14]. Соответствующий формализм используется ниже. Ограничимся построением нормализованных уравнений с точностью до кубических слагаемых включительно.

В п. 2.1 построена нормальная форма в случае $a_{0}=a_{1}=0$. В п. 2.2 рассмотрена повторная нормализация для этого случая. В п. 2.3 рассмотрен случай $a_{0}=0$, $a_{1}>0$. В п. 2.4 рассмотрен случай $a_{0}>0$. Сразу отметим, что результаты для этих трех случаев принципиально отличаются. 
2.1. Нормальная форма при $a_{0}=a_{1}=0$. В задаче $(9)$, (10) произведем перенормировку "времени" $\varepsilon t \rightarrow t$ и положим $y(t, x)=\varepsilon u(t, x)$. В результате получаем краевую задачу

$$
\begin{gathered}
\frac{\partial^{2} u}{\partial t^{2}}=\frac{\partial^{2} u}{\partial x^{2}}+\frac{\varepsilon^{2}}{12} \frac{\partial^{4} u}{\partial x^{4}}+\frac{2 \varepsilon^{4}}{6 !} \frac{\partial^{6} u}{\partial x^{6}}-b u^{3}, \\
u(t, x+2 \pi) \equiv u(t, x) .
\end{gathered}
$$

Линейное уравнение

$$
\frac{\partial^{2} u}{\partial t^{2}}=\frac{\partial^{2} u}{\partial x^{2}}+\frac{\varepsilon^{2}}{12} \frac{\partial^{4} u}{\partial x^{4}}+\frac{2 \varepsilon^{4}}{6 !} \frac{\partial^{6} u}{\partial x^{6}}
$$

с периодическими краевыми условиями (14) при $\varepsilon=0$ имеет совокупность периодических решений $u_{0}, \xi_{k} e^{i 2 \pi k(x+t)}, \eta_{k} e^{i 2 \pi k(x-t)}, k= \pm 1, \pm 2, \ldots$, где $u_{0}$ - вещественная, а $\xi_{k}$ и $\eta_{k}-$ комплексные постоянные. Согласно алгоритму из статей [10]-[12] для исследования решений нелинейной краевой задачи $(13),(14)$ введем в рассмотрение формальные ряды

$$
\begin{gathered}
u=\varepsilon\left(u_{0}(\tau)+\sum_{\substack{k=-\infty, k \neq 0}}^{+\infty} \xi_{k}(\tau) e^{i 2 \pi k(x+t)}+\sum_{\substack{k=-\infty \\
k \neq 0}}^{+\infty} \eta_{k}(\tau) e^{i 2 \pi k(x-t)}\right)+\varepsilon^{3} u_{3}(\tau, t, x)+\cdots, \\
\xi_{-k}(\tau)=\bar{\xi}_{k}(\tau), \quad \eta_{-k}(\tau)=\bar{\eta}_{k}(\tau) .
\end{gathered}
$$

Здесь $\tau=\varepsilon^{2} t-$ медленное время, зависимость от второго и третьего аргументов функций $u_{j}(\tau, t, x)$ является периодической. Еще раз отметим, что ряды вида (16) являются формальными, т. е. вопрос об их сходимости не рассматривается. Из результатов приводимых ниже утверждений следует, что они - асимптотические.

Формуле (16) можно придать более простой вид. Для этого положим

$$
\xi(\tau, x)=\sum_{\substack{k=-\infty \\ k \neq 0}}^{+\infty} \xi_{k}(\tau) e^{i 2 \pi k x}, \quad \eta(\tau, x)=\sum_{\substack{k=-\infty \\ k \neq 0}}^{+\infty} \eta_{k}(\tau) e^{i 2 \pi k x}
$$

Подставим выражения (16) в исходное уравнение (13). В получившемся формальном тождестве будем приравнивать коэффициенты при одинаковых степенях $\varepsilon$. Отметим, что переход от уравнения для функции $u$ к уравнениям для коэффициентов Фурье $u_{0}, \xi_{k}$ и $\eta_{k}$ называют нормализацией, а сами уравнения для новых медленных переменных - нормальной формой.

При первой степени $\varepsilon$ (в формальном тождестве) получаем верное равенство, а собирая коэффициенты при $\varepsilon^{3}$, приходим к краевой задаче для $u_{3}$

$$
\frac{\partial^{2} u_{3}}{\partial t^{2}}-\frac{\partial^{2} u_{3}}{\partial x^{2}}=F\left(u_{0}, \xi, \eta\right), \quad u_{3}(\tau, t, x+1) \equiv u_{3}(\tau, t, x),
$$

где

$$
\begin{gathered}
F\left(u_{0}, \xi, \eta\right)=-b\left(u_{0}+\xi+\eta\right)^{3}+2 \frac{\partial^{2} \xi}{\partial \tau \partial x}-2 \frac{\partial^{2} \eta}{\partial \tau \partial x}+\frac{1}{12}\left(\frac{\partial^{4} \xi}{\partial x^{4}}+\frac{\partial^{4} \eta}{\partial x^{4}}\right), \\
\xi=\xi(\tau, x+t), \quad \eta=\eta(\tau, x-t) .
\end{gathered}
$$


Для разрешимости уравнения (18) в указанном классе функций необходимо и достаточно, чтобы функция $F\left(u_{0}, \xi, \eta\right)$ не содержала гармоник $e^{i k(x+t)}$ и $e^{i k(x-t)}$ при $k=0, \pm 1, \ldots$. Применим этот критерий. В результате приходим к трем соотношениям. Во-первых, из условия равенства нулю коэффициента Фурье функции $F\left(u_{0}, \xi, \eta\right)$ при нулевой гармонике, т. е. из условия $M\left(F\left(u_{0}, \xi, \eta\right)\right)=0$, где принято обозначение $M(\varphi(x))=\int_{0}^{1} \varphi(x) d x$, получаем, что

$$
u_{0}^{3}+u_{0} A+B=0 .
$$

Здесь

$$
A=3\left(M\left(\xi^{2}\right)+M\left(\eta^{2}\right)\right), \quad B=3\left(M\left(\xi^{3}\right)+M\left(\eta^{3}\right)\right) .
$$

Во-вторых, из равенства нулю коэффициентов Фурье функции $F$ при гармониках $e^{i 2 \pi k(x+t)}, k= \pm 1, \pm 2, \ldots$, приходим к выводу, что

$$
2 \frac{\partial^{2} \xi}{\partial \tau \partial x}=\frac{1}{12} \frac{\partial^{4} \xi}{\partial x^{4}}+F_{1}\left(u_{0}, \xi, \eta\right)
$$

где $F_{1}\left(u_{0}, \xi, \eta\right)=3 b\left[u_{0}^{2} \xi+3 u_{0}\left(\xi^{2}-M\left(\xi^{2}\right)\right)+\xi^{3}-M\left(\xi^{3}\right)+3 M\left(\eta^{2}\right) \xi\right]$. Третье соотношение получается из приравнивания к нулю коэффициентов Фурье функции $F$ при гармониках $e^{i 2 \pi k(x-t)}, k= \pm 1, \pm 2, \ldots$ :

$$
-2 \frac{\partial^{2} \eta}{\partial \tau \partial x}=\frac{1}{12} \frac{\partial^{4} \eta}{\partial x^{4}}+F_{1}\left(u_{0}, \eta, \xi\right) .
$$

Отметим, что для $\xi$ и $\eta$ выполнены периодические краевые условия

$$
\xi(\tau, x+1) \equiv \xi(\tau, x), \quad \eta(\tau, x+1) \equiv \eta(\tau, x) .
$$

Поскольку значение $A$ в равенствах (20) положительно, то уравнение (19) относительно $u_{0}$ имеет единственное решение $u_{0}=u_{0}(\xi, \eta)$. Обратим внимание, что $u_{0}$ можно записать в виде

$$
u_{0}=v_{0}(\xi+\eta)
$$

причем функция $v_{0}(s)$ обладает свойством $v_{0}(c s)=c^{2} v_{0}(s)$, поскольку

$$
M\left(\xi^{2}\right)+M\left(\eta^{2}\right)=M\left((\xi+\eta)^{2}\right), \quad M\left(\xi^{3}\right)+M\left(\eta^{3}\right)=M\left((\xi+\eta)^{3}\right) .
$$

В силу этого нелинейность в выражениях (21), (22) является кубической.

Важно отметить, что "взаимодействие" уравнений (21) и (22) весьма специфично. В (21) влияние переменной $\eta$ осуществляется через усредненные величины $M\left(\eta^{2}\right)$ и $M\left(\eta^{3}\right)$, а в $(22)$ - через $M\left(\xi^{2}\right)$ и $M\left(\xi^{3}\right)$ соответственно.

Сформулируем результат о связи решений краевой задачи (13), (14) и решений (21)-(23). Его обоснование вытекает из приведенных выше построений.

Пусть $u_{0}, \xi_{0}(\tau, x+t)$ и $\eta_{0}(\tau, x-t)$ - решения краевой задачи $(19),(21)-(23)$. По ним из (18) можно найти функцию $u_{3}$. Легко проверить, что

$$
\begin{aligned}
u_{3}(\tau, t, x)=\frac{3 b}{4}[ & \left(2 u_{0} \eta_{0}+3\left(\eta_{0}^{2}-M\left(\eta_{0}^{2}\right)\right)\right) \int_{0}^{x+t} \xi_{0}(\tau, s) d s+ \\
& +\left(2 u_{0} \xi_{0}+3\left(\xi_{0}^{2}-M\left(\xi_{0}^{2}\right)\right)\right) \int_{0}^{x-t} \eta_{0}(\tau, s) d s+ \\
& \left.+\eta_{0} \int_{0}^{x+t}\left(\xi_{0}^{2}(\tau, s)-M\left(\xi_{0}^{2}\right)\right) d s+\xi_{0} \int_{0}^{x-t}\left(\eta_{0}^{2}(\tau, s)-M\left(\eta_{0}^{2}\right)\right) d s\right] .
\end{aligned}
$$


Теорема 1. Пусть выполнено условие $a_{0}=a_{1}=0$. Тогда краевая задача (13), (14) имеет асимптотическое по невязке с точностью до о $\left(\varepsilon^{3}\right)$ решение $u_{0}(t, x, \varepsilon)$, для которого

$$
u_{0}(t, x, \varepsilon)=\varepsilon\left(v_{0}\left(\xi_{0}+\eta_{0}\right)+\xi_{0}(\tau, x+t)+\eta_{0}(\tau, x-t)\right)+\varepsilon^{3} u_{3}(\tau, t, x) .
$$

2.2. Повторная нормализация при $a_{0}=a_{1}=0$. При учете в краевой задаче (21)-(24) слагаемых порядка $\varepsilon^{2}$ приходим к краевой задаче, которая отличается от (21)-(24) тем, что в правых частях уравнений (21) и (22) появляются соответственно слагаемые

$$
2 \varepsilon^{2}(6 !)^{-1} \frac{\partial^{6} \xi}{\partial x^{6}} \quad \text { и } \quad 2 \varepsilon^{2}(6 !)^{-1} \frac{\partial^{6} \eta}{\partial x^{6}},
$$

а также нелинейные слагаемые с множителем $\varepsilon^{2}$ пятого и выше порядков.

Для исследования поведения всех решений получившейся краевой задачи с начальными условиями из некоторой достаточно малой и независимой от $\varepsilon$ окрестности нулевого состояния равновесия применим схему исследования, использовавшуюся выше. Сначала рассмотрим линеаризованную краевую задачу. Поскольку уравнения для $\xi$ и $\eta$ похожи, то ограничимся тем, что приведем такое уравнение только для переменной $\xi$ :

$$
\frac{\partial^{2} \xi}{\partial \tau \partial x}=\frac{1}{12} \frac{\partial^{4} \xi}{\partial x^{4}}+\frac{2 \varepsilon^{2}}{6 !} \frac{\partial^{6} \xi}{\partial x^{6}}, \quad \xi(\tau, x+1) \equiv \xi(\tau, x) .
$$

Характеристическое уравнение для (27) имеет вид

$$
i 2 \pi k \lambda=\frac{(2 \pi k)^{4}}{12}-\frac{2 \varepsilon^{2}}{6 !}(2 \pi k)^{6}, \quad k= \pm 1, \pm 2, \pm 3, \ldots .
$$

В силу того, что все корни уравнения (28) являются чисто мнимыми, здесь снова реализуется критический случай бесконечной размерности в задаче об устойчивости. Решениями (27) являются периодические функции

$$
\exp \left[i 2 \pi k x-i\left(\left(\frac{2 \pi k}{12}\right)^{3}+\frac{2 \varepsilon^{2}}{6 !}(2 \pi k)^{5}\right) \tau\right], \quad k= \pm 1, \pm 2, \pm 3, \ldots
$$

Для исследования локальной динамики введем в рассмотрение формальные ряды

$$
\begin{aligned}
& \xi=\varepsilon \sum_{\substack{k=-\infty, p k \neq 0}}^{+\infty} z_{k}\left(\tau_{1}\right) \exp \left(i 2 \pi k x-\frac{i}{12}(2 \pi k)^{3} \tau\right)+\varepsilon^{3} z_{3}\left(\tau_{1}, \tau, x\right)+\cdots, \\
& \eta=\varepsilon \sum_{\substack{k=-\infty, k \neq 0}}^{+\infty} y_{k}\left(\tau_{1}\right) \exp \left(i 2 \pi k x+\frac{i}{12}(2 \pi k)^{3} \tau\right)+\varepsilon^{3} y_{3}\left(\tau_{1}, \tau, x\right)+\cdots
\end{aligned}
$$

Здесь $\tau_{1}=\varepsilon^{2} \tau-$ медленное время, $z_{-k}(\tau)=\bar{z}_{k}(\tau), y_{-k}(\tau)=\bar{y}_{k}(\tau)$, a $z_{j}\left(\tau_{1}, \tau, x\right)$ и $y_{j}\left(\tau_{1}, \tau, x\right)$ периодичны по $\tau$ и $x$. Подставим выражения $(29)$ и $(30)$ в $(21),(22)$ (с добавлением (26)) и соберем коэффициенты при $\varepsilon^{3}$. В результате получаем уравнения для $z_{3}$ и $y_{3}$. Условием их разрешимости в указанном классе функций является 
отсутствие в правых частях всех гармоник $e^{i 2 \pi k x \pm(i / 12)(2 \pi k)^{3} \tau}, k= \pm 1, \pm 2, \ldots$. В результате применения этого критерия приходим к следующей бесконечной системе обыкновенных дифференциальных уравнений для определения всех коэффициентов Фурье $z_{k}$ и $y_{k}$ :

$$
\begin{aligned}
2 \pi i k \frac{\partial z_{k}}{\partial \tau_{1}} & =\alpha(2 \pi k)^{6} z_{k}+F_{1 k}\left(v_{0}(\xi+\eta), \xi, \eta\right), \\
-2 \pi i k \frac{\partial y_{k}}{\partial \tau_{1}} & =\alpha(2 \pi k)^{6} y_{k}+F_{1 k}\left(v_{0}(\eta+\xi), \eta, \xi\right) .
\end{aligned}
$$

Здесь $\alpha=360^{-1}$, а через $F_{1 k}\left(v_{0}(\xi+\eta), \xi, \eta\right)$ обозначен коэффициент при $e^{i 2 \pi k x-i(2 \pi k)^{3} \tau / 12}$ разложения функции $F(v(\xi+\eta), \xi, \eta)$ при замене $\xi$ и $\eta$ соответственно на выражения

$$
\sum_{\substack{k=-\infty \\ k \neq 0}}^{+\infty} z_{k}\left(\tau_{1}\right) \exp \left(i 2 \pi k x-\frac{i}{12}(2 \pi k)^{3} \tau\right), \quad \sum_{\substack{k=-\infty \\ k \neq 0}}^{+\infty} y_{k}\left(\tau_{1}\right) \exp \left(i 2 \pi k x+\frac{i}{12}(2 \pi k)^{3} \tau\right)
$$

Предпримем попытку преобразовать бесконечномерную систему (31) к более компактному виду.

Напомним, что в предыдущем разделе выражение вида

$$
\sum_{\substack{k=-\infty \\ k \neq 0}}^{+\infty} \xi_{k}(\tau) e^{i 2 \pi k(x+t)}
$$

просто сворачивалось (см. формулу (17)) в $\xi(\tau, x+t)$. Тем самым $\xi_{k}(\tau)$ - соответствующий коэффициент Фурье. Для более сложных выражений (32) подобное сделать не удается. Поэтому поступим следующим образом.

Рассмотрим функции

$$
z(\tau, x)=\sum_{\substack{k=-\infty \\ k \neq 0}}^{+\infty} z_{k}(\tau) e^{i 2 \pi k x}, \quad y(\tau, x)=\sum_{\substack{k=-\infty \\ k \neq 0}}^{+\infty} y_{k}(\tau) e^{i 2 \pi k x}
$$

где коэффициенты Фурье $z_{k}(\tau)$ и $y_{k}(\tau)$ те же, что и в $(32)$. Здесь мы исходим из того, что, найдя $z\left(\tau_{1}, x\right)$ и $y\left(\tau_{1}, x\right)$, мы определяем все коэффициенты $z_{k}\left(\tau_{1}\right)$ и $y_{k}\left(\tau_{1}\right)$, а зная их, находим оба выражения (32). Обратим внимание, что при $\tau=\tau_{n}=12 n$, $n=1,2, \ldots$, выражения (32) совпадают соответственно с $z\left(\tau_{1}, x\right)$ и $y\left(\tau_{1}, x\right)$.

Введем обозначения. Пусть для некоторой функции $w(x)$ имеет место формула

$$
w(x)=\sum_{\substack{k=-\infty \\ k \neq 0}}^{+\infty} w_{k} e^{i 2 \pi k x}
$$

Через $N(w)$ обозначим бесконечномерный вектор

$$
N(w)=\left(\ldots, w_{-2} e^{-4 i \pi x}, w_{-1} e^{-2 i \pi x}, 0, w_{1} e^{2 i \pi x}, w_{2} e^{4 i \pi x}, \ldots\right) .
$$


Умножение двух бесконечномерных векторов будем считать покоординатным, т. е., для примера,

$$
N(w) \bar{N}(w)=\left(\ldots,\left|w_{-2}\right|^{2},\left|w_{-1}\right|^{2}, 0,\left|w_{1}\right|^{2},\left|w_{2}\right|^{2}, \ldots\right) .
$$

Тогда для скалярного произведения $(N(w), N(w) \bar{N}(w))$ имеем формулу

$$
(N(w), N(w) \bar{N}(w))=\sum_{\substack{k=-\infty \\ k \neq 0}}^{+\infty} w_{k}\left|w_{k}\right|^{2} e^{i 2 \pi k x}
$$

Рассмотрим краевую задачу

$$
\begin{array}{rlrl}
\frac{\partial^{2} z}{\partial \tau_{1} \partial x} & =\alpha \frac{\partial^{6} z}{\partial x^{6}}+F_{2}(z, y), & z\left(\tau_{1}, x+1\right) & \equiv z\left(\tau_{1}, x\right), \\
-\frac{\partial^{2} y}{\partial \tau_{1} \partial x}=\alpha \frac{\partial^{6} y}{\partial x^{6}}+F_{2}(y, z), & y(\tau, x+1) \equiv z(\tau, x),
\end{array}
$$

в которой

$$
F_{2}(z, y)=-9 b\left(2 z M\left(z^{2}\right)+z M\left(y^{2}\right)-3(N(z), N(z) \bar{N}(z))\right) .
$$

Сформулируем основной результат этого раздела.

ТЕОРЕма 2. Краевая задача (35) эквивалентна бесконечномерной системе уравнений (31).

Поясним сказанное: замена в (35) функций $z$ и y на ряды (33) приводит к той же самой системе (31).

Теорема 2 проверяется непосредственно подстановкой (33) в (35). Центральным моментом является отсутствие нетривиальных резонансов, т. е. для каждого фиксированного целого $k \neq 0$ система уравнений в целых и не равных нулю числах

$$
k_{1}+k_{2}+k_{3}=k, \quad k_{1}^{3}+k_{2}^{3}+k_{3}^{3}=k^{3}
$$

имеет только такие решения, где сумма двух каких-то чисел $k_{1}, k_{2}$ и $k_{3}$ равна нулю. Из теоремы 2 вытекает следующее утверждение.

Теорема 3. Пусть краевая задача (35) имеет ограниченное при $\tau_{1} \rightarrow \infty, x \in[0,1]$ решение $z_{0}\left(\tau_{1}, x\right), y_{0}\left(\tau_{1}, x\right)$ u

$$
z_{0}(\tau, x)=\sum_{\substack{k=-\infty, k \neq 0}}^{+\infty} z_{k_{0}}\left(\tau_{1}, x\right) e^{i 2 \pi k x}, \quad y_{0}(\tau, x)=\sum_{\substack{k=-\infty, k \neq 0}}^{+\infty} y_{k_{0}}\left(\tau_{1}, x\right) e^{i 2 \pi k x}
$$

Тогда краевая задача (21)-(23) имеет асимптотическое по невязке с точностъю до о $\left(\varepsilon^{3}\right)$ решение $\xi_{0}(\tau, x, \varepsilon), \eta_{0}(\tau, x, \varepsilon)$, для которого $\left(\right.$ при $\left.\tau_{1}=\varepsilon^{2} \tau\right)$

$$
\begin{aligned}
& \xi_{0}(\tau, x, \varepsilon)=\varepsilon \sum_{\substack{k=-\infty, k \neq 0}}^{+\infty} z_{k_{0}}\left(\tau_{1}, x\right) \exp \left(i 2 \pi k x-\frac{i}{12}(2 \pi k x)^{3} \tau\right)+\varepsilon^{3} z_{30}(\tau, x, \varepsilon), \\
& \eta_{0}(\tau, x, \varepsilon)=\varepsilon \sum_{\substack{k=-\infty, k \neq 0}}^{+\infty} y_{k_{0}}\left(\tau_{1}, x\right) \exp \left(i 2 \pi k x+\frac{i}{12}(2 \pi k x)^{3} \tau\right)+\varepsilon^{3} y_{30}(\tau, x, \varepsilon) .
\end{aligned}
$$


Краевую задачу (35) можно записать в несколько ином виде. Для этого введем оператор "интегрирования" $J(w)$ функции (34)

$$
J(w)=\sum_{\substack{k=-\infty, k \neq 0}}^{+\infty} \frac{w_{k}}{2 \pi i k} e^{i 2 \pi k x} .
$$

Тогда согласно (35) приходим к краевой задаче

$$
\begin{array}{rlrl}
\frac{\partial z}{\partial \tau_{1}} & =\alpha \frac{\partial^{5} z}{\partial x^{5}}+\Phi_{2}(z, y), & z\left(\tau_{1}, x+1\right) \equiv z(\tau, x), \\
-\frac{\partial y}{\partial \tau_{1}}=\alpha \frac{\partial^{5} y}{\partial x^{5}}+\Phi_{2}(y, z), & y\left(\tau_{1}, x+1\right) \equiv y(\tau, x),
\end{array}
$$

где

$$
\Phi_{2}(z, y)=-9 b\left[2 M\left(z^{2}\right) J(z)+M\left(y^{2}\right) J(z)-3(N(J(z)), N(z) \bar{N}(z))\right] .
$$

Поскольку характеристическое уравнение линеаризованной в нуле краевой задачи (36) имеет только чисто мнимые корни $\lambda_{k}=2 i(2 \pi k)^{5} / 6 !, k= \pm 1, \pm 2, \ldots$, и отсутствуют нетривиальные резонансы, то процесс нормализации можно продолжить. Действуя по аналогии с предыдущими построениями, в итоге приходим к краевой задаче

$$
\begin{aligned}
\frac{\partial^{2} v}{\partial \tau_{1} \partial x} & =F_{3}(v, w), & -\frac{\partial^{2} w}{\partial \tau_{1} \partial x} & =F_{3}(w, v), \\
v(\tau, x+1) & \equiv v(\tau, x), & w(\tau, x+1) & \equiv w(\tau, x),
\end{aligned}
$$

где $F_{3}(v, w)=-9 b\left[2 v M\left(v^{2}\right)+v M\left(w^{2}\right)-3(N(v), N(v) \bar{N}(v))\right]$.

Решения (36) связаны с решениями (37), (35) равенствами

$$
\begin{aligned}
& z=\sum_{\substack{k=-\infty, k \neq 0}}^{+\infty} v_{k}\left(\tau_{1}\right) e^{i 2 \pi k x-i \alpha(2 \pi k)^{6} \tau_{1}}+\cdots, \\
& y=\sum_{\substack{k=-\infty, k \neq 0}}^{+\infty} w_{k}\left(\tau_{1}\right) e^{i 2 \pi k x+i \alpha(2 \pi k)^{6} \tau_{1}}+\cdots
\end{aligned}
$$

Здесь через … обозначены слагаемые, которые являются асимптотически малыми по сравнению с предыдущим слагаемым, т. е. равны соответственно

$$
o\left(\sum_{\substack{k=-\infty, k \neq 0}}^{+\infty}\left|v_{k}\right|\right) \text { и } o\left(\sum_{\substack{k=-\infty, k \neq 0}}^{+\infty}\left|w_{k}\right|\right) .
$$

Подробнее на этом мы не останавливаемся.

2.3. Нормальная форма при $a_{0}=0, a_{1}>0$. Рассмотрим вопрос о поведении решений краевой задачи (13), (14) из малой окрестности нулевого состояния равновесия при условиях

$$
a_{0}=0, \quad a_{1}>0
$$


Линеаризованная в нуле краевая задача

$$
\frac{\partial^{2} u}{\partial t^{2}}=\frac{\partial^{2} u}{\partial x^{2}}+\frac{\varepsilon^{2}}{12} \frac{\partial^{4} u}{\partial x^{4}}+\frac{2 \varepsilon^{4}}{6 !} \frac{\partial^{6} u}{\partial x^{6}}-a_{1} u, \quad u(t, x+2 \pi) \equiv u(t, x),
$$

имеет характеристическое уравнение

$$
\lambda^{2}=-\left(k^{2}+a_{1}\right)+\frac{\varepsilon^{2}}{12} k^{4}-\frac{2 \varepsilon^{4}}{6 !} k^{6}, \quad k=0, \pm 1, \ldots
$$

Поскольку корни

$$
\lambda_{k}= \pm i\left(k^{2}+a_{1}-\frac{\varepsilon^{2}}{12} k^{4}+\frac{2 \varepsilon^{4}}{6 !} k^{6}\right)
$$

уравнения (41) являются чисто мнимыми, то (40) имеет периодические решения

$$
\begin{aligned}
& \alpha_{k} \exp \left(i k x-i t \sqrt{k^{2}+a_{1}-\frac{\varepsilon^{2}}{12} k^{4}+\frac{2 \varepsilon^{4}}{6 !} k^{6}}\right), \\
& \beta_{k} \exp \left(i k x+i t \sqrt{k^{2}+a_{1}-\frac{\varepsilon^{2}}{12} k^{4}+\frac{2 \varepsilon^{4}}{6 !} k^{6}}\right) .
\end{aligned}
$$

Согласно изложенной выше методике введем в рассмотрение формальный ряд

$$
u(t, x, \varepsilon)=\varepsilon u_{1}(\tau, t, x)+\varepsilon^{3} u_{3}(\tau, t, x)+\ldots,
$$

где

$$
\begin{aligned}
u_{1}(\tau, t, x)= & {\left[\varkappa(\tau)+\sum_{k=1}^{\infty} \xi_{k}(\tau) e^{i k x-i t \sqrt{k^{2}+a_{1}}}+\sum_{k=-1}^{-\infty} \xi_{k}(\tau) e^{i k x+i t \sqrt{k^{2}+a_{1}}}+\right.} \\
& \left.+\sum_{k=1}^{\infty} \eta_{k}(\tau) e^{i k x+i t \sqrt{k^{2}+a_{1}}}+\sum_{k=-1}^{-\infty} \eta_{k}(\tau) e^{i k x-i t \sqrt{k^{2}+a_{1}}}\right] .
\end{aligned}
$$

Здесь $\tau=\varepsilon^{2} t, \kappa(\tau)$ вещественна, $\xi_{-k}(\tau)=\bar{\xi}_{k}(\tau), \eta_{-k}(\tau)=\bar{\eta}_{k}(\tau)$, а функции $u_{j}(\tau, t, x)$ почти периодичны по второму и периодичны по третьему аргументам. Подставим (42) в (13). Применяя стандартные действия, приходим к уравнению для $u_{3}(\tau, t, x)$

$$
\frac{\partial^{2} u_{3}}{\partial t^{2}}-\frac{\partial^{2} u_{3}}{\partial x^{2}}+a_{1} u_{3}=F\left(u_{1}(\tau, t, x)\right)
$$

в котором

$$
F\left(u_{1}\right)=\frac{1}{12} \frac{\partial^{4} u_{1}}{\partial x^{4}}-b\left(u_{1}^{3}\right)
$$

Это уравнение разрешимо при условии отсутствия в разложении $F\left(u_{1}\right)$ гармоник $e^{i k x \pm i t \sqrt{k^{2}+a_{1}}}, k=0, \pm 1, \ldots$. Отсюда получаем, что $\varkappa(\tau) \equiv 0$,

$$
\begin{aligned}
-i \sqrt{k^{2}+a_{1}} \frac{\partial^{2} \xi_{k}}{\partial \tau^{2}} & =\frac{k^{4}}{12} \xi_{k}+F_{k 1}, \\
i \sqrt{k^{2}+a_{1}} \frac{\partial^{2} \eta_{k}}{\partial \tau^{2}} & =\frac{k^{4}}{12} \eta_{k}+F_{k 2}
\end{aligned}
$$


где $F_{k 1}$ и $F_{k 2}$ - соответствующие коэффициенты Фурье функции $F\left(u_{1}\right)$. Обозначим через $\mathbb{L}$ банахово пространство, получающееся замыканием по норме $C$ множества всех тригонометрических многочленов, элементами которых являются функции $e^{ \pm i t \sqrt{k^{2}+a_{1}}}, k=0, \pm 1, \ldots$. Бесконечную систему уравнений (44) можно записать в виде

$$
\begin{aligned}
\frac{\partial^{2} \xi}{\partial \tau \partial y}= & \frac{1}{12}\left[\frac{\partial^{4} \xi}{\partial y^{4}}-2 a_{1} \frac{\partial^{2} \xi}{\partial y^{2}}+2 a_{1}^{2} \xi\right]- \\
& -9 b\left(2 \xi M(\xi)^{2}+\xi\left(M\left(\eta^{2}\right)\right)-3(N(\xi), N(\xi) \bar{N}(\xi))\right) \\
-\frac{\partial^{2} \xi}{\partial \tau \partial y}= & \frac{1}{12}\left[\frac{\partial^{4} \xi}{\partial y^{4}}-2 a_{1} \frac{\partial^{2} \xi}{\partial y^{2}}+2 a_{1}^{2} \xi\right]- \\
& -9 b\left(2 \eta M\left(\eta^{2}\right)+\eta\left(M\left(\xi^{2}\right)\right)-3(N(\eta), N(\eta) \bar{N}(\eta))\right)
\end{aligned}
$$

где $\xi, \eta \in \mathbb{L}$.

Важный вывод заключается в том, что система (45) играет роль нормальной формы для краевой задачи (13), (14) при условии (39).

Теорема 4. Пусть система (45) имеет ограниченное при $\tau \rightarrow \infty$ решение $\xi(\tau, y)$, $\eta(\tau, y)$ (из пространства $\mathbb{L}$ по переменной $y)$. Тогда краевая задача (13), (14) имеет асимптотическое по невязке с точностью до о $\left(\varepsilon^{3}\right)$ решение $u_{0}(t, y, \varepsilon)$, для которого

$$
u_{0}(t, y, \varepsilon)=\varepsilon u_{1}(\tau, t, x)+\varepsilon^{3} u_{03}(\tau, t, x) .
$$

Отметим, что можно выписать явно все коэффициенты Фурье функции $u_{03}(\tau, t, x)$ из уравнения (43). Обратим внимание, что при $x=0$ (и $x=2 \pi$ ) имеем равенство

$$
u_{0}(t, 0, \varepsilon)=\varepsilon\left(\xi_{0}(\tau, t)+\eta_{0}(\tau, t)\right)+o\left(\varepsilon^{3}\right) .
$$

ДокАзАТЕЛьство. Подставим формальный ряд (42) в (13). Как оказывается, в получающейся приравниванием коэффициентов при $\varepsilon^{3}$ бесконечномерной системе обыкновенных дифференциальных уравнений относительно $\xi_{k}$ и $\eta_{k}$ отсутствуют нетривиальные резонансы.

Сформулируем соответствующее утверждение.

ЛЕмма 1. Система уравнений в различных цельх числах $k_{1}, k_{2}, k_{3}$

$$
\begin{gathered}
k_{1}+k_{2}+k_{3}=k, \\
\sqrt{k_{1}^{2}+a_{1}}+\sqrt{k_{2}^{2}+a_{1}}+\sqrt{k_{3}^{2}+a_{1}}=\sqrt{k^{2}+a_{1}}
\end{gathered}
$$

для каждого иелого $k$ не имеет решений, а система

$$
\begin{aligned}
k_{1}+k_{2} & =k_{3}+k \\
\sqrt{k_{1}^{2}+a_{1}}+\sqrt{k_{2}^{2}+a_{1}} & =\sqrt{k_{3}^{2}+a_{1}}+\sqrt{k^{2}+a_{1}}
\end{aligned}
$$

имеет только такие решения, для которых (с точностью до переобозначений) $k_{1}=k_{3}, k_{2}=k$. 
Относительно системы (46) утверждение очевидно, а для обоснования соответствующего утверждения для системы (47) введем обозначения. Положим

$$
k_{1}+k_{2}=A, \quad k_{1} k_{2}=B_{1}, \quad k_{3} k=B_{2} .
$$

Тогда из первого уравнения в (47), возводя в квадрат левую и правую части, получаем, что

$$
k_{1}^{2}+k_{2}^{2}=A^{2}-2 B_{1}, \quad k_{3}^{2}+k^{2}=A^{2}-2 B_{2} .
$$

Возведем в квадрат левую и правую части второго уравнения в (47). Тогда с учетом (49) получаем, что

$$
f\left(B_{1}\right)=f\left(B_{2}\right),
$$

где $f(B)=A^{2}-2 B+2 a+2 \sqrt{B^{2}+a\left(A^{2}-2 B\right)+a^{2}}$. Отметим, что $f^{\prime}(B)<0$, поэтому функция $f(B)$ монотонна, а значит, согласно равенству (50) приходим к выводу, что $B_{1}=B_{2}$. Отсюда и на основании (48) завершаем обоснование леммы.

Для завершения доказательства теоремы 4 достаточно в (45) учесть разложение в соответствующие ряды Фурье пространства $\mathbb{L}$ функций $\xi$ и $\eta$ и убедиться, что для коэффициентов Фурье $\xi_{k}$ и $\eta_{k}$ мы получаем ту же самую систему (44).

В порядке обсуждения полученных здесь результатов заметим, что условие $\xi, \eta \in \mathbb{L}$ в (45) ведет к усложнению исследования этой системы. Если бы в левых частях (44) вместо множителя $\sqrt{k^{2}+a_{1}}$ стоял множитель $k$, то вместо (45) мы получили бы краевую задачу

$$
\begin{aligned}
-\frac{\partial^{2} \xi}{\partial \tau \partial x} & =\frac{1}{12} \frac{\partial^{4} \xi}{\partial x^{4}}-9 b\left(2 \xi M\left(\xi^{2}\right)+\xi M\left(\eta^{2}\right)-3(N(\xi), N(\xi) \bar{N}(\xi))\right), \\
\frac{\partial^{2} \eta}{\partial \tau \partial x} & =\frac{1}{12} \frac{\partial^{4} \eta}{\partial x^{4}}-9 b\left(2 \eta M\left(\eta^{2}\right)+\eta M\left(\xi^{2}\right)-3(N(\eta), N(\eta) \bar{N}(\eta))\right),
\end{aligned}
$$

$\xi(\tau, x+2 \pi) \equiv \xi(\tau, x), \eta(\tau, x+2 \pi) \equiv \eta(\tau, x)$.

Возможно, для асимптотического изучения решений системы (44) предпочтительнее использовать вместо системы (45) систему (51) с заменой $\sqrt{k^{2}+a_{1}}$ в левых частях (44) на $k$ только при достаточно больших значениях $|k|$. Если же номер $|k|$ нельзя считать достаточно большим, то для таких номеров (а их, очевидно, конечное число) следует использовать в качестве базовой систему (44).

2.4. Нормальная форма при $a_{0}>0$. Более интересна ситуация, когда

$$
a_{0}>0
$$

В этом случае при $\varepsilon=0$ характеристическое уравнение (6) имеет пару чисто мнимых корней $\pm i a$, а значит, (5) имеет при $\varepsilon=0$ периодические решения $\xi e^{ \pm i a_{0} t}$. Для построения асимптотических разложений решений $(9),(10)$ введем, следуя методике работ [9]-[12], формальный ряд

$$
y=\varepsilon\left[\xi(\tau, x) e^{i a t}+\bar{\xi}(\tau, x) e^{-i a t}\right]+\varepsilon^{3} y_{3}(\tau, t, x)+\cdots,
$$

где $\tau=\varepsilon^{2} t$, а функции $y_{j}(\tau, t, x)$ периодичны по второму и третьему аргументам. Подставляя (53) в задачу (5), приходим к уравнению для определения $\xi(\tau, x)$

$$
2 i a_{0} \frac{\partial \xi}{\partial \tau}=\frac{\partial^{2} \xi}{\partial x^{2}}-\left(a_{1}+3 b|\xi|^{2}\right) \xi
$$


с периодическими краевыми условиями

$$
\xi(\tau, x+2 \pi) \equiv \xi(\tau, x) .
$$

Отметим, что уравнение (54) является классическим уравнением Шредингера. Решения этого уравнения дают возможность получить асимптотические по невязке решения уравнения (9) согласно формуле (53). В частности, периодическому решению в (54), (55) соответствует тор в (9), (10). Интересные результаты о поведении решений при больших временах уравнений вида (54), а также результаты об интегрируемости и существовании точного решения уравнения (54) приведены в работах [15], [17], [18].

\section{3. БЫСТРО ОСЦИЛЛИРУЮЩИЕ РЕШЕНИЯ}

Решения вида (8) формируются в основном на "невысоких" модах, т. е. на таких, которые соответствуют конечным при $\varepsilon \rightarrow 0$ значениям $k$ из (6). В этом разделе мы рассматриваем вопрос о нерегулярных решениях (3), (4), формирующихся на асимптотически высоких (при $\varepsilon \rightarrow 0$ ) модах.

Зафиксируем произвольно параметр $\delta \neq 0$ и будем исследовать решения (3), (4), формирующиеся на модах с номерами

$$
k= \pm\left(2 \delta \varepsilon^{-1}+\theta+m\right) .
$$

Здесь величина $\theta=\theta(\varepsilon) \in[0,1)$ дополняет в (56) предыдущее слагаемое до целого, $m=0, \pm 1, \ldots$ При таких номерах $k$ для корней $\lambda=\lambda_{m}$ в (6) имеем представление

$$
\lambda_{m}= \pm i\left[\gamma(\delta)\left(1+\frac{\varepsilon(\theta+m) \sin 2 \delta}{2 \gamma^{2}(\delta)}\right)+O\left(\varepsilon^{2}\right)\right],
$$

где $\gamma(\delta)=\sqrt{4 \sin ^{2} \delta+a_{0}^{2}}$.

Введем в рассмотрение формальный ряд

$$
\begin{aligned}
y= & \varepsilon\left[\sum_{m=-\infty}^{+\infty} \xi_{m}(\tau) \exp \left\{i\left(\frac{2 \delta}{\varepsilon}+\theta+m\right) x+i \gamma(\delta)\left(1+\frac{\varepsilon(\theta+m) \sin 2 \delta}{2 \gamma^{2}(\delta)}+o\left(\varepsilon^{2}\right)\right) t\right\}+\right. \\
& \left.+\sum_{m=-\infty}^{+\infty} \eta_{m}(\tau) \exp \left\{i\left(\frac{2 \delta}{\varepsilon}+\theta+m\right) x-i \gamma(\delta)\left(1+\frac{\varepsilon(\theta+m) \sin 2 \delta}{2 \gamma^{2}(\delta)}+o\left(\varepsilon^{2}\right)\right) t\right\}+\overline{c c}\right]+ \\
& +\varepsilon^{3} y_{3}(\tau, x, t)+\cdots .
\end{aligned}
$$

Здесь $\tau=\varepsilon^{2} t$, через $\overline{c c}$ обозначаются слагаемые, комплексно сопряженные к предыдущим, а $y_{j}$ периодически зависят от $x$ и от $t$.

Введем обозначения. Положим

$$
\xi(\tau, x)=\sum_{m=-\infty}^{+\infty} \xi_{m}(\tau) e^{i m x}, \quad \eta(\tau, x)=\sum_{m=-\infty}^{+\infty} \eta_{m}(\tau) e^{i m x} .
$$

Тогда формула (58) принимает вид

$$
y=\varepsilon y_{0}(\tau, t, x)+\varepsilon^{3} y_{3}(\tau, t, x)+\cdots,
$$


где

$$
\begin{aligned}
y_{0}(\tau, t, x)= & \xi\left(\tau, z_{+}\right) \exp \left(i\left(\frac{2 \delta}{\varepsilon}+\theta\right) x+i \gamma(\delta)\left(1+\varepsilon \theta \gamma^{-2}(\delta) 2 \sin 2 \delta\right) t\right)+ \\
& +\eta\left(\tau, z_{-}\right) \exp \left(i\left(\frac{2 \delta}{\varepsilon}+\theta\right) x-i \gamma(\delta)\left(1+\varepsilon \theta \gamma^{-2}(\delta) 2 \sin 2 \delta\right) t\right)+\overline{c c}
\end{aligned}
$$

$z_{ \pm}=x \pm \varepsilon \gamma^{-1} \delta t \sin 2 \delta$. Подставляя выражение (59) в (3) и производя стандартные действия, для определения неизвестных амплитуд $\xi(\tau, x)$ и $\eta(\tau, x)$ получаем систему уравнений

$$
L^{+}(\delta) \xi=-3 b \xi\left[|\xi|^{2}+2|\eta|^{2}\right], \quad L^{-}(\delta) \eta=-3 b \eta\left[2|\xi|^{2}+|\eta|^{2}\right]
$$

с периодическими краевыми условиями

$$
\xi\left(\tau, z_{+}+2 \pi\right) \equiv \xi\left(\tau, z_{+}\right), \quad \eta\left(\tau, z_{-}+2 \pi\right) \equiv \eta\left(\tau, z_{-}\right) .
$$

Здесь

$$
\begin{gathered}
L^{ \pm}(\delta) w= \pm\left(2 i \gamma(\delta) \frac{\partial w}{\partial \tau}\right)-R(\delta)\left[\frac{\partial^{2} w}{\partial z_{ \pm}^{2}}+4 i \theta \frac{\partial w}{\partial z_{ \pm}}-4 \theta^{2} w\right]-2 a_{0} a_{1} w \\
R(\delta)=\cos 2 \delta-\gamma^{-2}(\delta) \sin 2 \delta
\end{gathered}
$$

Отметим, что при $a_{0}=0$ имеем $\gamma(\delta)=-2 \sin \delta$ и $R(\delta)=-\sin ^{2} \delta$. Связь между решениями краевой задачи (60), (61) и исходной краевой задачи $(3),(4)$ устанавливает следующее утверждение. В нем фигурирует последовательность $\varepsilon_{n} \rightarrow 0$, которая определяется условием $\theta(\varepsilon)=\theta_{0}$.

ТЕОРема 5. Зафиксируем произвольно параметры $\delta$ и $\theta_{0} \in[0,1)$. Пусть $\xi\left(\tau, z_{+}\right)$ и $\eta\left(\tau, z_{-}\right)$- решения (60), (61) при $\theta=\theta_{0}$, ограниченные $п р и \tau \rightarrow \infty, x \in[0,2 \pi]$. Тогда при $\varepsilon=\varepsilon_{n}$ краевая задача (3), (4) имеет асимптотическое по невязке с точностью до о $\left(\varepsilon^{3}\right)$ решение $\widetilde{y}\left(t, x, \varepsilon_{n}\right)$, для которого имеет место представление (59)

$$
\widetilde{y}(t, x, \varepsilon)=\varepsilon y_{0}(\tau, t, x)+\varepsilon^{3} y_{3}(\tau, t, x) .
$$

Напомним, что переход от исходных уравнений к уравнениям для медленно меняющихся амплитуд называют нормализацией, а сами уравнения - нормальными, а иногда - квазинормальными формами. В этом смысле семейство уравнений шредингеровского типа (60), (61), зависящее от параметров $\delta$ и $\theta$, является квазинормальной формой.

\section{1. Взаимодействующие системы быстро осциллирующих решений.} Зафиксируем произвольно номер $N$ и различные положительные значения $\delta_{1}, \ldots, \delta_{N}$. Через $\theta_{j}=\theta_{j}(\varepsilon) \in[0,1)$ обозначим величину, дополняющую выражение $\delta_{j} \varepsilon^{-1}$ до целого.

Основное упрощающее предположение относительно величин $\delta_{j}$ состоит в том, что отсутствуют нетривиальные резонансы, т. е. системы уравнений

$$
\begin{aligned}
\delta_{j_{1}}+\delta_{j_{2}} \pm \delta_{j_{3}} & =\delta_{j}, \\
\gamma\left(\delta_{j_{1}}\right)+\gamma\left(\delta_{j_{2}}\right) \pm \gamma\left(\delta_{j_{3}}\right) & =\gamma\left(\delta_{j}\right)
\end{aligned}
$$


могут иметь только такие решения, для которых одно из слагаемых в левой части (62) совпадает со слагаемым в правой части этого же уравнения.

Решения (3), (4) будем искать в виде формального ряда

$$
\begin{aligned}
y=\varepsilon( & \sum_{j=1}^{N}\left(\xi_{j}\left(\tau, z_{j+}\right) \exp \left[i\left(\delta_{j} \varepsilon^{-1}+\theta_{j}\right) x+i \gamma\left(\delta_{j}\right)\left(1+\varepsilon \theta_{j} \gamma^{-2}\left(\delta_{j}\right) \sin 2 \delta_{j}\right) t\right]+\right. \\
& \left.\left.+\eta_{j}\left(\tau, z_{j-}\right) \exp \left[i\left(\delta_{j} \varepsilon^{-1}+\theta_{j}\right) x-i \gamma\left(\delta_{j}\right)\left(1+\varepsilon \theta_{j} \gamma^{-2}\left(\delta_{j}\right) \sin 2 \delta_{j}\right) t\right]+\overline{c c}\right)\right)+ \\
& +\varepsilon^{3} y_{3}+\cdots,
\end{aligned}
$$

где $z_{j \pm}=x \pm \varepsilon \gamma^{-1} t \sin 2 \delta_{j}$. Подставим ряд (64) в формулу (3). После стандартных процедур приходим к системе краевых задач относительно неизвестных амплитуд $\xi_{j}$ и $\eta_{j}$

$$
\begin{aligned}
& L^{+}\left(\delta_{j}\right) \xi_{j}=-3 b \xi_{j}\left(2 \sum_{k=1}^{N}\left(\left|\xi_{k}\right|^{2}+\left|\eta_{k}\right|^{2}\right)-\left|\xi_{j}\right|^{2}\right), \\
& L^{-}\left(\delta_{j}\right) \eta_{j}=-3 b \eta_{j}\left(2 \sum_{k=1}^{N}\left(\left|\xi_{k}\right|^{2}+\left|\eta_{k}\right|^{2}\right)-\left|\eta_{j}\right|^{2}\right)
\end{aligned}
$$

с периодическими краевыми условиями

$$
\xi_{j}\left(\tau, z_{j_{+}}+2 \pi\right) \equiv \xi_{j}\left(\tau, z_{j_{+}}\right), \quad \eta_{j}\left(\tau, z_{j_{-}}+2 \pi\right) \equiv \eta_{j}\left(\tau, z_{j_{-}}\right) .
$$

\section{2. Пространственно распределенные в двумерной области системы.} В отличие от предыдущих пунктов, здесь мы изучаем случай бесконечного множества значений $\delta_{n}$, например когда $\delta_{n}=n \delta, n=0, \pm 1, \ldots$ В ситуации, когда на параметр $\delta$ не накладывается никаких ограничений, получить уравнения, записанные в компактной форме, не удается. Ниже относительно параметра $\delta$ предполагаем, что для некоторых натуральных взаимно простых чисел $m_{1}$ и $m_{2}$ и для произвольного фиксированного $\alpha>0$ выполнено равенство $\delta=2 m_{1} m_{2}^{-1} \pi+\varepsilon \alpha$. Будем исследовать такие решения (3), которые формируются на модах с номерами

$$
k= \pm\left[\left(\frac{2 m_{1} \pi}{\varepsilon m_{2}}+\alpha+\theta\right) n+m\right], \quad n, m=0, \pm 1, \ldots,
$$

где $\theta=\theta(\varepsilon) \in[0,1)$ дополняет до целого сумму двух предыдущих слагаемых в (65). Тогда для корней $\lambda(n, m)$ в (6) верно равенство

$$
\lambda(n, m)= \pm i\left[\gamma\left(\frac{2 m_{1} \pi}{m_{2}} n\right)\left\{1+\varepsilon[(\theta+\alpha) n+m] \sin \left(\frac{m_{1} \pi}{m_{2}} n\right)\right\}\left\{2 \gamma^{2}\left(\frac{m_{1} \pi}{m_{2}} n\right)\right\}^{-1}+o\left(\varepsilon^{2}\right)\right] .
$$

Заметим, что каждое из значений $n$ можно представить в виде $n=m_{2} r+j$, где $r=0, \pm 1, \ldots, j=1, \ldots, m_{2}$. Поэтому выражение (66) можно так разбить на $m_{2}$ группы $\lambda_{j}(n, m), j=1, \ldots, m_{2}$, что

$$
\begin{aligned}
\lambda(n, m)= & \pm i\left[\gamma\left(\frac{2 m_{1} j \pi}{m_{2}}\right)\left(1+\varepsilon(\theta+\alpha)\left(m_{2} n+j+m\right) \sin \left(\frac{2 m_{1} j \pi}{m_{2}}\right)\right) \times\right. \\
& \left.\times\left(2 \gamma^{2}\left(\frac{m_{1} j \pi}{m_{2}}\right)\right)^{-1}+o\left(\varepsilon^{2}\right)\right], \quad n, m=0, \pm 1, \ldots
\end{aligned}
$$


Тогда аналогом выражений (58) и (59) являются формальные ряды

$$
\begin{aligned}
y=\varepsilon( & \sum_{j=1}^{m_{2}} \xi_{j}\left(\tau, v_{j_{+}}, z_{j_{+}}\right) \exp \left[i m_{2} \varepsilon v_{j_{+}}+i \gamma\left(\frac{2 m_{1} j \pi}{m_{2}}\right) t\right]+ \\
& \left.+\sum_{j=1}^{m_{2}} \eta_{j}\left(\tau, v_{j_{-}}, z_{j_{-}}\right) \exp \left[i m_{2} \varepsilon v_{j_{-}}-i \gamma\left(\frac{2 m_{1} j \pi}{m_{2}}\right) t\right]\right)+\varepsilon^{3} y_{3}+\cdots
\end{aligned}
$$

Здесь

$$
\begin{gathered}
\tau=\varepsilon^{2} t, \quad v_{j_{ \pm}}=\left(\frac{2 m_{1} j \pi}{m_{2} \varepsilon}+\theta_{j}\right) x \pm \varepsilon(\alpha+\theta)\left(\frac{2 m_{1} j \pi}{m_{2}}\right) t \\
z_{j_{ \pm}}=x \pm \varepsilon \gamma^{-1}\left(\frac{2 m_{1} j \pi}{m_{2}}\right) \sin \left(\frac{4 m_{1} j \pi}{m_{2}}\right) t
\end{gathered}
$$

а функция $y_{3}(\tau, v, z), z=\left(z_{1_{ \pm}}, \ldots, z_{m_{2 \pm}}\right), v=\left(v_{1_{ \pm}}, \ldots, v_{m_{2}}\right)$, периодична по всем элементам $v_{j_{ \pm}}$и $z_{j_{ \pm}}$.

Подставляя ряды (68) в (3) и производя стандартные действия, для определения неизвестных амплитуд $\xi_{j}$ и $\eta_{j}$ получаем специальные системы уравнений.

Сначала остановимся на двух наиболее простых случаях.

Пусть $m_{1}=1, m_{2}=2$. Тогда

$$
k= \pm\left[\left(\frac{\pi}{\varepsilon}+\alpha+\theta\right) n+m\right], \quad n, m=0, \pm 1, \ldots
$$

Здесь $v=(\pi / \varepsilon+\alpha+\theta) x$, а для $y$ имеем асимптотическое представление

$$
y=\varepsilon\left(\xi(\tau, v, x) e^{i a_{0} t}+\bar{\xi}(\tau, v, x) e^{-i a_{0} t}\right)+\varepsilon^{3} y_{3}(\tau, v, x)+\cdots,
$$

причем зависимость от $v$ и $x$ в (70) является периодической. Для $\xi(\tau, v, x)$ приходим к краевой задаче следующего вида.

Случай 1. Для $a_{0}=0$ имеем $\tau=\varepsilon t$ и

$$
\begin{aligned}
& \frac{\partial^{2} \xi}{\partial \tau^{2}}=\left(\frac{\partial}{\partial x}+\frac{(\alpha+\theta)}{2 \pi} \frac{\partial}{\partial v}\right)^{2} \xi-a_{1} \xi-b \xi^{3}, \\
& \xi(\tau, v+1, x) \equiv \xi(\tau, v, x) \equiv \xi(\tau, v, x+2 \pi) .
\end{aligned}
$$

Случай 2. Для $a_{0} \neq 0$ имеем $\tau=\varepsilon^{3} t$ и получаем двумерное уравнение Шредингера

$$
i a_{0} \frac{\partial \xi}{\partial \tau}=\left(\frac{\partial}{\partial x}+\frac{\alpha+\theta}{2 \pi} \frac{\partial}{\partial v}\right)^{2} \xi-a_{1} \xi-3 b \xi|\xi|^{2}
$$

с краевыми условиями (72).

Положим затем $m_{1}=1, m_{2}=4$ и пусть

$$
k= \pm\left[\left(\frac{\pi}{2 \varepsilon}+\alpha+\theta\right)(2 n+1)+m\right], \quad n, m=0, \pm 1, \ldots .
$$

В этом случае при $\tau=\varepsilon^{2} t$ приходим к уравнению

$$
2 i \sqrt{4+a_{0}^{2}} \frac{\partial \xi}{\partial \tau}=\left(\frac{\partial}{\partial x}+\frac{\alpha+\theta}{\pi} \frac{\partial}{\partial v}\right)^{2} \xi-a_{1} \xi-3 b \xi|\xi|^{2}
$$


с 1-антипериодическими по $v$ и $2 \pi$-периодическими по $x$ краевыми условиями

$$
-\xi(\tau, v+1, x) \equiv \xi(\tau, v, x) \equiv \xi(\tau, v, x+2 \pi) .
$$

Функция $y$ связана с $\xi(\tau, v, x)$ равенством

$$
y=\varepsilon\left(\xi(\tau, v, x) \exp \left(i t \sqrt{4+a_{0}^{2}}\right)+\bar{\xi}(\tau, v, x) \exp \left(-i t \sqrt{4+a_{0}^{2}}\right)+\overline{c c}\right)+\varepsilon^{3} y_{3}+\cdots .
$$

Предположим затем, что $k= \pm((\pi / 2 \varepsilon+\alpha+\theta) n+m), n, m=0, \pm 1, \ldots$. Тем самым при четных $n$ мы попадаем в условие (69), а при нечетных $n$ - в условие (74). Для простоты ограничимся здесь случаем $a_{0} \neq 0$.

Для решения $y$ имеем асимптотическое представление

$$
y=\varepsilon\left(\left(\xi_{1}(\tau, v, x) \exp \left(i t a_{0}\right)+\xi_{2}(\tau, v, x) \exp \left(i t \sqrt{4+a_{0}^{2}}\right)+\overline{c c}\right)+\varepsilon^{3} y_{3}+\cdots,\right.
$$

а для определения $\xi_{j}(\tau, v, x)$ приходим к системе уравнений

$$
\begin{aligned}
2 i a_{0} \frac{\partial \xi_{1}}{\partial \tau} & =\left(\frac{\partial}{\partial x}+(\alpha+\theta) \frac{\partial}{\partial v}\right)^{2} \xi_{1}-a_{1} \xi_{1}-3 b \xi_{1}\left(\left|\xi_{1}\right|^{2}+2\left|\xi_{2}\right|^{2}\right), \\
i \sqrt{4+a_{0}^{2}} \frac{\partial \xi_{2}}{\partial \tau} & =\left(\frac{\partial}{\partial x}+(\alpha+\theta) \frac{\partial}{\partial v}\right)^{2} \xi_{2}-a_{1} \xi_{2}-3 b \xi_{2}\left(\left|\xi_{2}\right|^{2}+2\left|\xi_{1}\right|^{2}\right) .
\end{aligned}
$$

Для $\xi_{1}$ и $\xi_{2}$ выполнены краевые условия $(72)$ и (75) соответственно.

Новые интересные моменты возникают при условии $\delta \neq \pi n / 2$. Для примера рассмотрим случай, когда $m_{1}=1, m_{2}=3$ и

$$
k= \pm\left[\left(\frac{2 \pi}{3 \varepsilon}+\alpha+\theta\right)(3 n+1)+m\right], \quad n, m=0, \pm 1, \ldots .
$$

Здесь

$$
\begin{aligned}
y=\varepsilon[ & \xi\left(\tau, v_{+}, z_{+}\right) \exp \left(i\left(\frac{2 \pi}{3 \varepsilon}+\alpha+\theta\right) x+\sqrt{3+a_{0}^{2}}\left(1+\varepsilon \frac{\sqrt{3}}{2\left(3+a_{0}^{2}\right)}(\alpha+\theta)\right) t\right)+ \\
& +\eta\left(\tau, v_{-}, z_{-}\right) \exp \left(i\left(\frac{2 \pi}{3 \varepsilon}+\alpha+\theta\right) x-\sqrt{3+a_{0}^{2}}\left(1+\varepsilon \frac{\sqrt{3}}{2\left(3+a_{0}^{2}\right)}(\alpha+\theta)\right) t\right)+ \\
& +\overline{c c}]+\varepsilon^{3} y(\tau, v, z)+\cdots,
\end{aligned}
$$

где

$$
v=\left(1+\frac{3 \varepsilon(\alpha+\theta)}{2 \pi}\right) \frac{x}{\varepsilon} \pm \frac{3 \sqrt{3} \varepsilon(\alpha+\theta)}{4 \pi \sqrt{3+a_{0}^{2}}} t, \quad z_{ \pm}=x \pm \frac{\sqrt{3} \varepsilon}{2 \sqrt{3+a_{0}^{2}}} t,
$$

а $y$ периодически зависит от $v$ и $x$. В итоге для $\xi(\tau, v, x)$ приходим к краевой задаче

$$
\begin{gathered}
i\left(3+a_{0}^{2}\right) \frac{\partial \xi}{\partial \tau}=A\left(\frac{\alpha+\theta}{2 \pi} \frac{\partial}{\partial v_{+}}+\frac{\partial}{\partial z_{+}}\right)^{2} \xi-\frac{a_{1}}{3+a_{0}^{2}} \xi-3 b \xi\left(|\xi|^{2}+2|\eta|^{2}\right), \\
-i\left(3+a_{0}^{2}\right) \frac{\partial \eta}{\partial \tau}=A\left(\frac{\alpha+\theta}{2 \pi} \frac{\partial}{\partial v_{-}}+\frac{\partial}{\partial z_{-}}\right)^{2} \eta-\frac{a_{1}}{3+a_{0}^{2}} \eta-3 b \eta\left(|\eta|^{2}+2|\xi|^{2}\right), \\
\xi\left(\tau, v_{+}+1, z_{+}\right) \equiv \xi\left(\tau, v_{+}, z_{+}\right) \equiv \xi\left(\tau, v_{+}, z_{+}+2 \pi\right), \\
\eta\left(\tau, v_{-}+1, z_{-}\right) \equiv \eta\left(\tau, v_{-}, z_{-}\right) \equiv \eta\left(\tau, v_{-}, z_{-}+2 \pi\right) .
\end{gathered}
$$

Здесь $A=8^{-1}\left(3+a_{0}^{2}\right)^{-1 / 2}\left(a_{0}^{2}(\sqrt{3}-1)+6 \sqrt{3}\right)$. 


\section{4. ВЫВОДЫ}

Рассмотрены асимптотические по невязке решения исходного дифференциального уравнения с отклонениями пространственной переменной. В различных областях из малой окрестности нулевого состояния равновесия фазового пространства построены специальные нелинейные распределенные уравнения и системы уравнений, зависящие от континуальных семейств некоторых параметров. В частности показано, что решения исходного пространственного одномерного уравнения можно описать решениями специальных уравнений и систем уравнений шредингеровского типа в пространственно двумерной области изменения аргументов.

На первый взгляд может показаться, что получившиеся нормальные формы не проще или даже сложнее исходных уравнений. Но это не так. Суть раскрывают теоремы $1-5$. Они говорят о том, что главная часть решений уравнения (3) является решением именно нормализованных уравнений. Таким образом, решая исходные уравнения, приходится не только находить главные части решений, но и вычислять еще ряд довольно сложных функций. Кроме этого важно иметь в виду, что нормальные формы содержат существенно меньше по сравнению с первоначальной задачей гармоник Фурье. Все это говорит о том, что нормальные формы могут существенно помочь в изучении исходной задачи.

Отметим, что только нормальные формы из раздела 1 являются таковыми в традиционном понимании этого термина как системы уравнений для определения медленно меняющихся амплитуд. Повторные нормальные формы из п. 2.2 и нормальные формы из п. 2.3, вообще говоря, таковыми не являются, но по их решениям можно эффективно построить и медленно меняющиеся амплитуды, и сами решения исходной задачи.

Из построенных нормальных форм следуют важные выводы о взаимодействии волн, движущихся в противоположных направлениях, т. е. о влиянии переменных $\xi$ и $\eta$ друг на друга. Самое главное - это влияние является интегральным: действие одной переменной на другую определяется средним по пространственной переменной квадрата амплитуды $\xi$ или $\eta$. Интересно отметить, что в формально близкой задаче Ферми-Паста-Улама ситуация иная. Там взаимодействие приводит лишь к сдвигу фазовых скоростей [9].

\section{Список литературы}

[1] Я.И. Френкель, Т. А. Конторова, "К теории пластической деформации и двойникования. I", ЖЭТФ, 8 (1938), 89-97; "К теории пластической деформации и двойникования. II", 1340-1348; "K теории пластической деформации и двойникования. III", $1349-1358$.

[2] Ч. Уэрт, Р. Томсон, Физика твердого тела, Мир, М., 1969.

[3] E. Fermi, J. Pasta, S. Ulam, Studies of Nonlinear Problems. I, Report LA-1940, Los Alamos Scientific Laboratory of the University of California, Los Alamos, 1955.

[4] T. Genta, A. Giorgilli, S. Paleari, T. Penati, "Packets of resonant modes in the Fermi-Pasta-Ulam system", Phys. Lett. A, 376:28-29 (2012), 2038-2044.

[5] Н.А. Кудряшов, Аналитическая теория нелинейных дифференииальных уравнений, ИКИ, М.-Ижевск, 2004.

[6] C.S. Gardner, J. M. Greene, M.D. Kruskal, R. M. Miura, "Method for solving the Korteweg-deVries equation", Phys. Rev. Lett., 19:19 (1967), 1095-1097.

[7] М. Абловиц, Х. Сигур, Солитонъ и метод обратной задачи, Мир, М., 1987. 
[8] N. A. Kudryashov, "From the Fermi-Pasta-Ulam model to higher-order nonlinear evolution equations", Rep. Math. Phys., 77:1 (2016), 57-67.

[9] Д. С. Глызин, С.А. Кащенко, А. О. Толбей, "Взаимодействие двух волн в модели Ферми-Паста-Улама", Модел. и анализ информ. систем, 23:5 (2016), 548-558.

[10] С. А. Кащенко, "Нормальная форма для уравнения Кортевега-де Фриза-Бюргерса", Докл. РАН, 468:4 (2016), 383-386.

[11] S. A. Kaschenko, "Normalization in the systems with small diffusion", Internat. J. Bifur. Chaos, 6:6 (1996), 1093-1109.

[12] I. S. Kaschenko, S. A. Kaschenko, "Local dynamics of the two-component singular perturbed systems of parabolic type", Internat. J. Bifur. Chaos, 25:11 (2015), 1550142, 27 pp.

[13] С. А. Кащенко, "О квазинормальных формах для параболических уравнений с малой диффузией", Докл. АН СССР, 299:5 (1988), 1049-1052.

[14] И. С. Кащенко, С. А. Кащенко, "Квазинормальные формы двухкомпонентных сингулярно возмущенных систем", Докл. РАН, 447:4 (2012), 376-381.

[15] Н. А. Кудряшов, Методы нелинейной математической физики, "Интеллект", Долгопрудный, 2010.

[16] N. A. Kudryashov, "Analytical properties of nonlinear dislocation equation", Appl. Math. Lett., 69 (2017), 29-34.

[17] П. И. Наумкин, "Асимптотика при больших временах решений нелинейного уравнения Шрёдингера", Изв. РАН. Сер. матем., 61:4 (1997), 81-118.

[18] П. И. Наумкин, "Диссипативное свойство кубического нелинейного уравнения Шрёдингера", Изв. РАН. Сер. матем., 79:2 (2015), 137-166.

Поступила в редакцию 19.06.2017, после доработки 29.08.2017 